\title{
Beam Me Out: Review of Emergency Department Telepsychiatry and Lessons Learned During COVID-19
}

\author{
Nabil Natafgi ${ }^{1}\left(\right.$ ) Casey Childers ${ }^{2,3} \cdot$ Amanda Pollak $^{2,3} \cdot$ Shanikque Blackwell ${ }^{1} \cdot$ Suzanne Hardeman ${ }^{2,3}$. \\ Stewart Cooner ${ }^{4} \cdot$ Robert Bank $^{4} \cdot$ Brenda Ratliff $^{4} \cdot$ Victoria Gooch $^{4} \cdot$ Kenneth Rogers $^{4} \cdot$ Meera Narasimhan $^{2,3}$
}

Accepted: 28 July 2021 / Published online: 6 October 2021

(c) The Author(s), under exclusive licence to Springer Science+Business Media, LLC, part of Springer Nature 2021

\begin{abstract}
Purpose of Review The aim of this review is to critically evaluate recent literature on the use of telepsychiatry in emergency departments (EDTP) and synthesize the evidence on telepsychiatry during public health emergencies. We also report on experiences and success stories from a state-wide EDTP program in South Carolina during the COVID-19 pandemic.

Recent Findings We identified 12 peer-reviewed articles published between January 2019 and February 2021 that evaluated EDTP interventions and their impact on patient outcomes. The recent evidence on EDTP shows a significant association between EDTP implementation or use and decreased patients' wait time in emergency department (ED), shorter length of stay in certain settings, reduced ED revisit rates, improved ED patient disposition (e.g., more discharge to home, less observational stays, and decreased inpatient admissions), and reduced follow-up encounters involving self-harm diagnosis. Summary The EDTP virtual delivery model can help healthcare systems reduce burden of public health emergencies on providers, staff, and patients alike. While a disruption of magnitude seen by COVID-19 may be infrequent, strategies used during the pandemic may be implemented to enhance care in rural settings, and/or enhance preparedness of communities and healthcare systems during more commonly occurring natural disasters.
\end{abstract}

Key Words Emergency telepsychiatry $\cdot$ Mental health $\cdot$ Telemedicine $\cdot$ Telehealth $\cdot$ Emergency department $\cdot$ Public health emergency

\section{Introduction}

Telepsychiatry provides remote mental health care services for clinical non-urgent encounters, as well as emergencies, such as urgent care or public health emergencies involving disasters and pandemics like coronavirus disease 2019

This article is part of the Topical Collection on Psychiatry in the Digital Age

Suzanne Hardeman

Suzanne.Hardeman@uscmed.sc.edu

1 Health Services Policy \& Management, Arnold School of Public Health, University of South Carolina, Columbia, SC, USA

2 Department of Neuropsychiatry and Behavioral Science, School of Medicine, University of South Carolina, Columbia, SC, USA

3 Prisma Health - Midlands, SC, Columbia, USA

4 South Carolina Department of Mental Health, Columbia, SC, USA
(COVID-19). Telepsychiatry has the potential to address challenges encountered during emergencies and improve patient care in a cost-effective manner [1-4]. While its nationwide deployment has been historically restricted, telepsychiatry proved effective and satisfactory to both emergency department staff and the patients with mental health conditions [5]. This evolving technology can reduce emergency department (ED) overcrowding, expand access to vital care in rural areas, and provide access to psychiatric care during and after disasters or emergencies [5]. A recent review of literature concluded that ED-based telepsychiatric consultations were associated with "reduction of length of stay, drop in inpatient admissions, increased cost-effectiveness, and improved satisfaction of patients and staff" [6••]. Even though telemedicine is now more frequently integrated into emergency medical response planning, there has been limited use of telemedicine in national emergencies situations prior to 2020 [7].

Since the height of COVID-19, healthcare utilization witnessed significant changes in the way patients seek care. ED psychiatry was one of many specialties that initially saw a 
significant decline in patient utilization once the state of public health emergency was declared, with trends reflected nationally as well as globally [8-14]. In 2020, overall ED usage in the USA dropped considerably, averaging about a $42 \%$ drop from the year prior [15]. Globally, similar trends were observed. In Portugal, one study found a 52\% decrease in the number of in-person psychiatric emergency visits during the second quarter of 2020, compared to the same period in 2019 [16]. These reductions in ED visits have translated to fewer assessments being conducted with fewer diagnoses of mood disorders [9]. Further, rate of admission for psychiatric ED patients was slightly higher than previous years [14], suggesting a delay in care-seeking until urgently needed. It is believed that the ED utilization declines do not represent a reduced prevalence of illness in the population, but a deferral of seeking care [14]. This led to rapid adoption of telepsychiatry by EDs, to address many of the COVID-19-related challenges, including personal protective equipment shortages, limited staffing, patient and staff safety, and physical distancing requirements.

The aim of this article is twofold. First, we provide a synthesis of the literature on telepsychiatry over the last 2 years with a specific focus on telepsychiatry use in ED settings, especially during COVID-19. Second, we present observations from the field, exploring the stakeholder experiences of a state-wide ED-based Telepsychiatry (EDTP) Program during COVID-19. We conclude with lessons learned and implications on clinical psychiatry practice and mental health policy.

\section{Methods}

We searched PubMed and Google Scholar for peer-reviewed original research studies and other published reports on any telepsychiatric intervention in the ED setting published between January 2019 and February 2021. For PubMed, the following MeSH search terms were used: "Remote Consultation," "Telemedicine," and "Videoconferencing" in combination with "Mental Disorders," "Mental Health," "Mental Health Services," "Psychotherapy," and "Psychiatry" in combination with "Crisis Intervention," "Emergency Service, Hospital" and "Emergency Services, Psychiatric." In addition, an expanded list of free text terms using [tiab] field code after each term was used in PubMed (the list available upon request). For Google Scholar, the following search terms were used: "telepsychiatry," "tele-mental health," "videoconferencing," and "teleconsultation" in combination with "emergency department," "emergency room." We checked the reference section of each included study to identify other potential studies. We also reviewed grey (nonpeer-reviewed literature) using the same terms on the regular Google search engine to identify case studies or unpublished success stories during COVID-19.
We included only studies that examined outcomes or addressed lessons learned from a telemedicine or telehealth intervention targeting patients with any psychiatric or mental health condition in an ED setting. Non-empirical articles were not included in the summary table but used to inform lessons learned, recommendations, and policy implications. Only studies published in English were reviewed and included. Two members of the research team independently reviewed the titles, abstracts, and full text to decide on article inclusion and abstract key elements and information from the articles. A third team member reviewed both inclusion lists and abstracted material, consolidated the lists, and resolved any discrepancies.

\section{Results}

A national survey of 5375 EDs in the USA in 2016, by Freeman and colleagues, showed that 885 (20\%) of the 4507 EDs

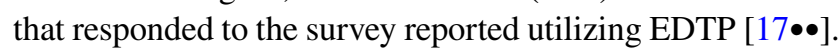
In this review, we identified 12 peer-reviewed articles that evaluated or discussed telepsychiatry services or telehealth interventions for mental health services in emergency or ED settings (Table 1).

\section{The Impact of EDTP on Patient Outcomes}

Since the review by Reinhardt and colleagues in 2019 [6••], seven more recent studies addressed the use of EDTPs, further substantiating previous evidence on telepsychiatry efficiency. For example, Freeman et al. showed that EDTP decreased the wait time for patient transfer and departure from ED to inpatient bed from 1 to 30 days to around $12 \mathrm{~h}$ [17••]. Similarly, Reliford and colleagues showed that EDTP reduced total length of stay (LOS) for patients in pediatric ED [18•]. Fairchild and colleagues found similar results for telehealth consultation in rural EDs for behavioral health cases: average ED wait time for the telehealth visits was shorter at $12 \mathrm{~min}$ (95\% CI 11-14 $\mathrm{min}$ ) compared to average time of $27 \mathrm{~min}$ (95\% CI 22-32 $\mathrm{min}$ ) for the non-telehealth visits [18•]. However, in the same study, LOS for the EDTP visits in rural EDs was significantly longer $(\mathrm{M}=318 \mathrm{~min}$ vs. $147 \mathrm{~min}, p<0.001$ ) compared to the non-EDTP visits [19]. Implementing psychiatric assessment through EDTP has also shown to improve rural ED efficiency and reduce recidivism (i.e., up to $36 \%$ lower all-cause ED revisit rate within 90 days) by improving the competence of local ED healthcare providers to assess, treat, and manage patients through weekly telementoring experiences with a psychiatric specialist [20].

Kothadia et al. examined ED discharge records for 86,931 patients with psychiatric symptoms to study the impact of 


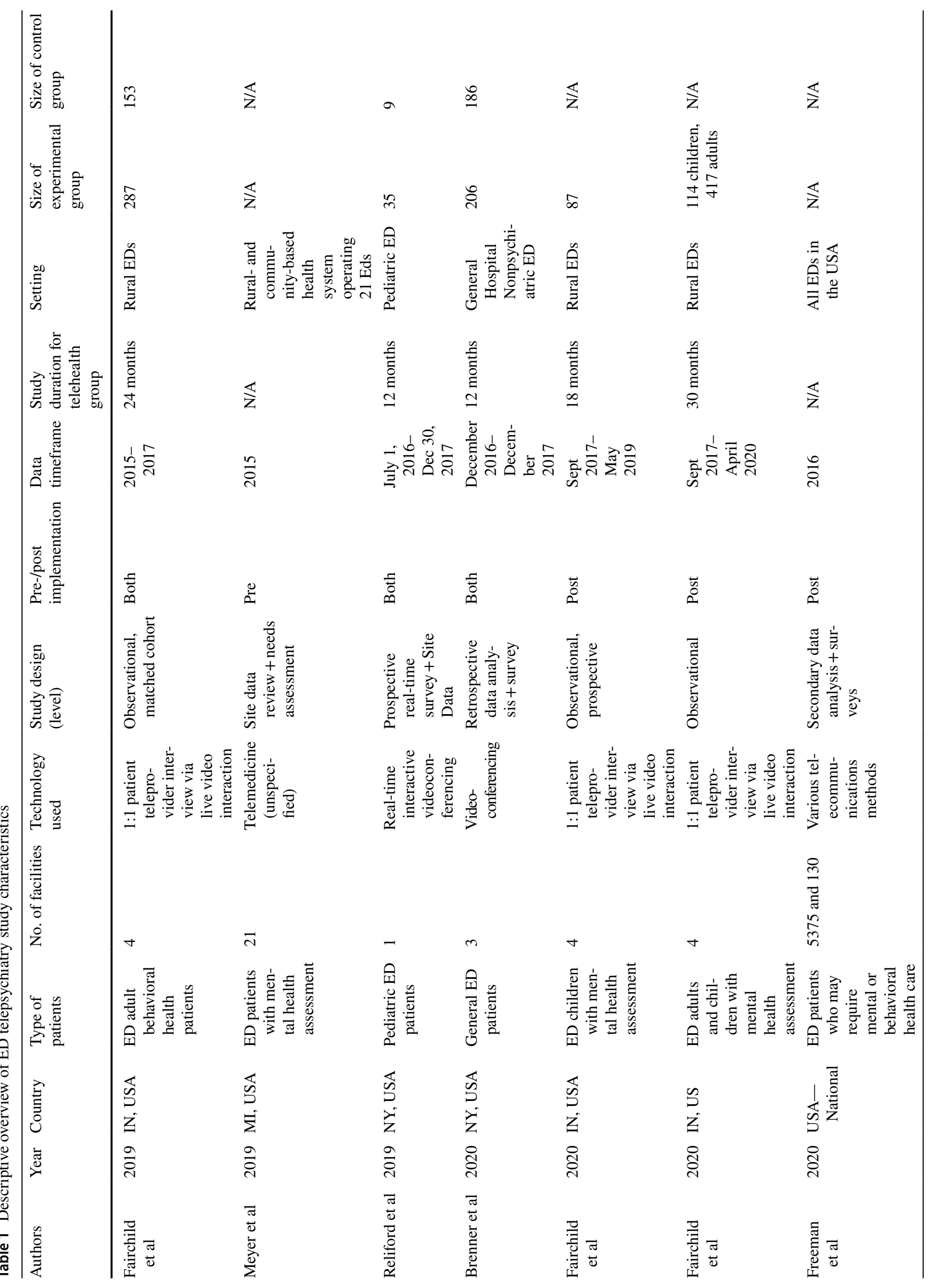




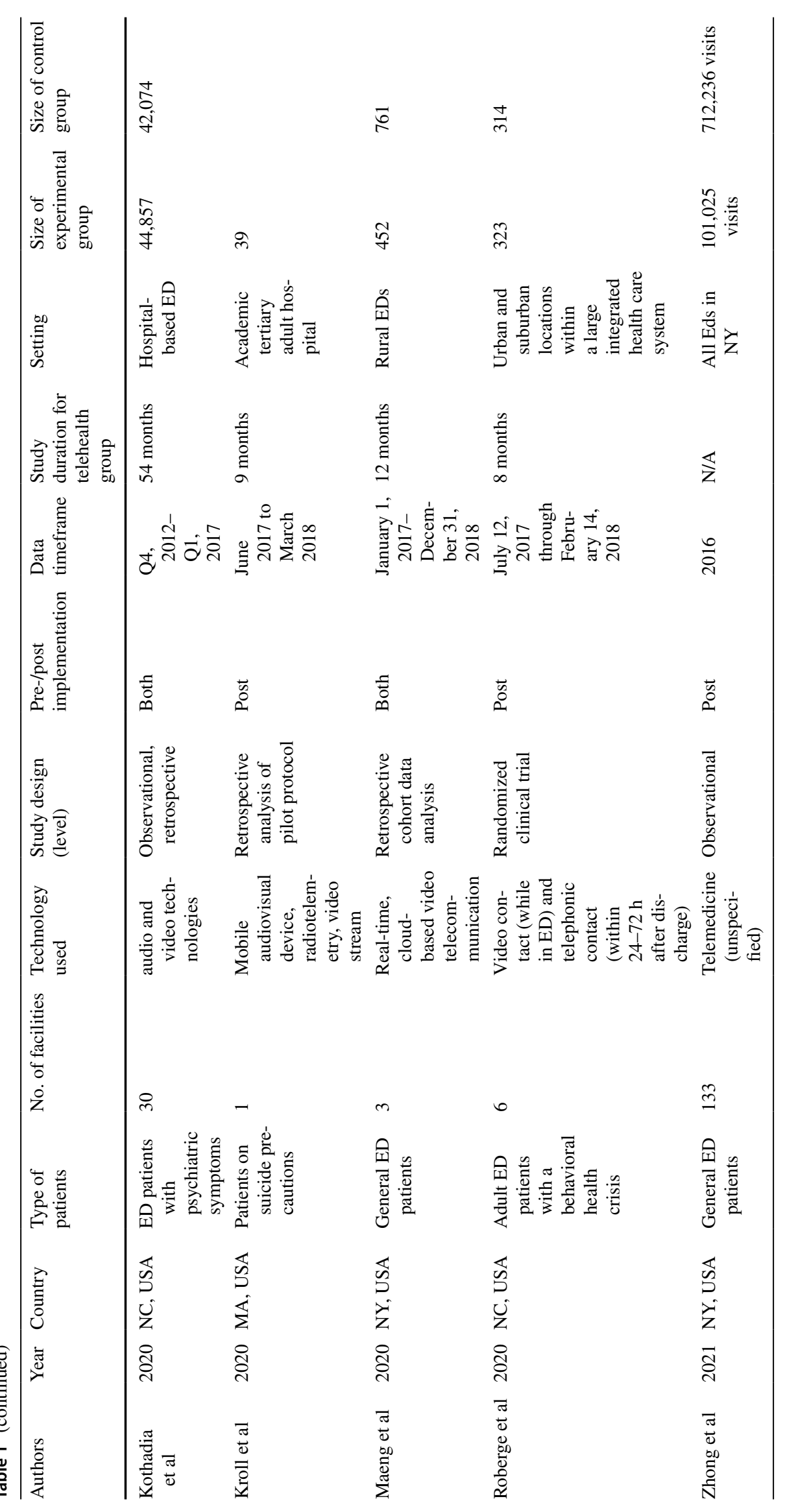


a statewide telepsychiatry program in North Carolina (NCSTeP) on ED patient disposition and LOS [21]. They found that when utilizing telepsychiatry, the rate of discharge and transfers increased as compared to patients that did not receive telepsychiatry consults [21]. Furthermore, EDs were also able to discharge about $62 \%$ of those with extended LOS as compared to only $43 \%$ being discharged home prior to the NC-STeP implementation [21].

Zhong and colleagues shed a different light on ED efficiency by assessing the impact of observation visits (stays of 8-48 h) on disposition across a larger cohort of psychiatric visits to EDs with and without EDTPs by linking the 2016 National ED Inventory-USA to psychiatric visits from the 2016 New York State ED Databases/State Inpatient Database [22]. They demonstrated that psychiatric visits in EDTPequipped EDs used less observational services (2.3\% of ED visits) compared to those without EDTP capacity (2.7\%) with adjusted odds ratio 0.30 and concluded that receipt of EDTP was associated with "lower usage of observation services for psychiatric visits, likely reducing the amount of time spent in the ED and mitigating the ongoing problem of ED crowding" [22]. There is also evidence that the implementation of a virtual patient navigation program in six EDTP-equipped EDs spanning urban and suburban locations across North Carolina, South Carolina, and Georgia decreased the admission rate for patients with mental health outcomes and reduced the number of follow-up encounters involving self-harm diagnosis within 45 days [23•].

\section{Provider and Patients/User Experiences}

Despite certain barriers to patients with specific conditions, most patients typically agree that telepsychiatry - in general - is just as good as in-person, with a high percentage of patients expressing satisfaction with telepsychiatry care [24-26]. However, some providers expressed difficulty with reading nonverbal communication cues, requiring increased efforts to build rapport [25•]. Providers also expressed logistical challenges such as private space, learning how to troubleshoot equipment issues, and navigating new clinic-patient dynamics, as well as concerns about payment and productivity (e.g., feelings fatigue) [25•]. Further, one study argued although telepsychiatry programs have support from staff and patients and can facilitate virtual staff meeting, clinical rounds, and consultations, they may also increase the time burden on nursing staff [27].

Six of the twelve included studies assessed provider and/ or patient/other user experiences with EDTP interventions. Brenner et al. demonstrated that $97 \%$ of the patients surveyed were satisfied with the EDTP services provided in non-psychiatric EDs [24]. Two studies of an EDTP intervention in rural EDs by Fairchild et al. argued that telepsychiatry was efficacious, allowing patients to have increased access, enhanced responsiveness, and better expertise from their care teams [28, 29]. Meyer et al. also surveyed ED providers in a rural- and community-based health system operating 21 EDs (none of which has direct access to psychiatric consultation) and reported that "on a rating scale of 0-100 with 0 representing 'Very Uncomfortable' and 100 representing 'Very Comfortable,' the average reported comfort level with the concept of ED telepsychiatry consultation was 80" [30]. Kroll et al. evaluated a virtual monitoring intervention that uses video technology to assess suicide risk of patients in EDs and determined that no adverse events were noted among the patients being monitored virtually [31]. In addition, medical residents felt telepsychiatry decreased travel time for face-to-face evaluation, while improving the amount of time that passed from consultation to evaluation [18•].

\section{Telepsychiatry Use During Public Health Emergencies like COVID-19}

Telepsychiatry can be an effective strategy in bridging service and access gaps, both as a supplement in areas where psychiatric services may be limited and during widescale disruptions or public health emergencies, such as pandemics or natural disasters. The expansion of telepsychiatry during the COVID-19 pandemic built on the technology's strong evidence base, demonstrating its effectiveness for various psychiatric treatments, populations, and settings [1-4, 32-35]. The rapid establishment of robust outpatient services via telepsychiatry may have preserved or even expanded access to treatment for many patients [14]. According to the Centers for Disease Control and Prevention (CDC), the first quarter of 2020 had a 50\% increase in the number of overall telehealth visits compared with the same period in 2019 [36]. Most recently, around $30 \%$ of weekly health center visits occurred via telehealth between June and November 2020 [37].

While it was previously used to enhance access to mental health services during and after disasters [38], telepsychiatry's use in the COVID-19 pandemic is unique and expected to have long-lasting and wide-ranging impact on the field of psychiatry. For instance, 54\% of patients using telepsychiatry surveyed immediately after the stay-at-home order in Michigan stated that they would continue with virtual care even after in-person visits resume [39•]. Patients reasoning for preference for virtual visits included convenience, lower chances of infection, and provider availability, while those preferring in-person visits cited lack of comfort with digital technology as their primary motivation [39•].

The rapid transition to telemedicine during COVID-19 was partly facilitated by several federal, state, and local policies that reduced restrictions and enhanced coverage and payment to psychiatric telehealth services, reduced prior authorization requirements, enhanced Medicaid coverage, and allowed 
90-day prescriptions with automatic refills [40, 41]. Other telepsychiatry advantages highlighted by COVID-19 and its policy changes include assisting with current staffing burdens while attracting new mental health professionals who may not have previously considered working in telepsychiatry. Also, telepsychiatry allowed for new procedures around patient and staff safety, increased social connection previously denied because of restrictive policies, and increased real-time responsiveness [42].

While these new policies improved access, they may have created a new set of barriers to vulnerable population on issues such as technology access and literacy hurdles [43]. This calls for more research to understand the risk and benefits (including cost-effectiveness) of this telepsychiatry and EDTP as well as any ethical issues specifically during a public health emergency [42]. It is worth noting that despite several peer-reviewed evidence on telemedicine in general and telepsychiatry during COVID-19, we did not retrieve any article in the past year that focused on telepsychiatry in the ED setting during COVID-19.

\section{South Carolina EDTP Program Experiences During COVID-19}

South Carolina is home to a robust statewide EDTP program, which has been empirically shown to improve access to mental health care along with value and quality of care [44]. While a more extensive description of the program can be found elsewhere [44], the protocol notably uses a statewide queue. Participating EDs enter patients' name and information into the queue, which is then handled by an available pool of ED telepsychiatrists located statewide. During COVID-19, the existing established network of EDTP providers served as an advantage by ensuring an available pool of personnel as ED telepsychiatrists could be re-assigned as needed, while limiting provider exposure to COVID-19.

We asked four stakeholders who represent the South Carolina Department of Mental Health (SCDMH) ED Telepsychiatry Program (SC-EDTP) about first-hand experiences and direct observations during COVID-19. The SCDMH stakeholders were asked about their direct observations regarding trends seen in South Carolina's EDs before and during the COVID-19 pandemic, with a focus on how SC-EDTP enabled healthcare systems to meet the needs of patients during a truly historical phenomenon. SCDMH stakeholder experiences were included to help fill the current literature gap on ED telepsychiatry programs during COVID-19.

\section{SCDMH and SC-EDTP Program Infrastructure}

The SCDMH is one of the largest direct mental health care providers in the country, serving approximately 100,000 patients per year [45]. There are 16 community mental health centers (CMHCs) with satellite offices serving all $46 \mathrm{SC}$ counties, three licensed hospitals, and four nursing homes [45]. In addition to SC-EDTP, SCDMH programming includes a statewide mobile crisis program for mental health emergencies and the Community Telepsychiatry program, which allows SCDMH patients to receive care from a psychiatrist located in another county when the local SCDMH $\mathrm{CMHC}$ does not have a psychiatrist physically present.

SCDMH stakeholders reported that one of the key advantages of SC-EDTP was it connects EDs to CMHCs and resources in the community, creating continuity to outpatient care. Additionally, having a shared electronic health record between SC-EDTP and CMHCs links the patient information from crisis care to outpatient care. SC-EDTP physicians can also communicate directly with the CMHC program directors about high-risk patients so that CMHCs are prepared to get the patients in the programs swiftly.

After 14 years of the program, there are still hospitals that do not have access to a psychiatrist and are not part of SCEDTP, according to the interviewed stakeholders. Hospitals have barriers that are not necessarily related to the size of the hospital or funding, but more so operating in highly isolated areas where resources are limited, especially to mental health care. The group also mentioned the SC-EDTP patient payer mix as an important factor in program viability. For SC hospitals with larger groups of patients with no reimbursements, it is difficult to employ a psychiatrist to service patients at the hospital, especially after hours. However, SC-EDTP is exploring how to serve hospitals with different levels of funding and capacity.

Even with the successes of SC-EDTP, SCDMH stakeholders reported the program still requires supplemental funding, despite having the ability for all SC-EDTP physicians to bill for services since the beginning of the program. This is due to the high number of uninsured patients, at approximately $70 \%$. SC-EDTP received funds from The Duke Endowment in early phases of the program, and later from the South Carolina General Assembly and the South Carolina Telehealth Alliance.

\section{SC-EDTP During COVID-19}

The SCDMH stakeholders reported a decrease in consultation requests for the SC-EDTP program at the beginning of COVID-19, starting in April 2020. SC-EDTP volume preCOVID-19 was approximately 620 per month for completed requests, with completed requests dipping April-September 2020 to approximately 490 per month. The group attributed this decrease to people not seeking ED treatment due to personal safety concerns with COVID-19 or the perception that EDs were being overwhelmed. Nonetheless, SC-EDTP volume returned to pre-COVID-19 volume after September 2020 . 
Patient acuity level increased during the beginning of the COVID-19 pandemic. Before COVID-19, most SC-EDTP patients could be stabilized and discharged from the ED. However, most patients in spring 2020 had to be admitted and were manic, psychotic, and severely ill. The interviewed stakeholders suggested two reasons: (i) more people who did not have mental health issues before COVID-19 were being treated for mental health issues in the ED, or (ii) only the most severely ill patients sought treatment in the ED for mental health issues. Another aspect that changed during COVID-19 was patient age. There were very few children treated in spring 2020; however, the numbers rose and have continued to rise. In addition to the higher number of child patients being treated in SC-EDTP, the acuity level is higher. The stakeholders reported having half of the entire SC-EDTP physician workforce trained in child and adolescent care helped to deal with the child patient increase.

We've had a lot of kids for the last 2 weeks, amazingly young kids...most were under 10 . So, we had some really young kids presenting and really sick kids.

One factor that helped the SC-EDTP staff with changes during COVID-19 was continuing monthly SC-EDTP staffing meetings, even if there were few agenda items discussed. While the meetings were focused on patients and problemsolving, SCDMH stakeholders reported this meeting was also a listening session and helped physicians cope with living through the pandemic, both as mental health professionals and in personal lives.

I think just the support of being able to talk about things and have time to talk to them as a group helped because it's pretty isolating.....and you're not seeing or talking to another doctor.... We talked about the changes that were occurring.

When COVID-19 began, several hundred SCDMH outpatient specialists shifted from in-person care models to providing direct outpatient services from their homes within a matter of weeks. The SCDMH stakeholders agreed that before COVID-19, expanded direct to patient telehealth services was not the SCDMH plan. However, since telepsychiatry was part of the SCDMH infrastructure, platforms shifted quickly. Getting people access to high-quality mental health care in the outpatient setting has improved continuity of care, treatment adherence, and reduced missed appointments, which can lead to gaps in prescription refills.

Thank God we knew how to do telepsychiatry because it went way beyond the ED. We were ready [for COVID-19].

In the end, the group thinks SCDMH, as an organization, will learn from the positives and the negatives of telehealth during a crisis. It is unclear if SCDMH will continue the expanded telehealth programs or expand further. SCDMH stakeholders mentioned Centers for Medicare and Medicaid Services (CMS) rules as a key factor in these decisions.

A lot of us in the telehealth arena are looking to see what CMS is going to pay once the public health emergency ends. So, while the loosening of restrictions has allowed telemedicine to expand dramatically, including the types of services provided and then the types of providers, everybody's looking to see what they are going to pay for when this public health emergency ends. That's really going to define what the landscape looks like going forward.

\section{Discussion}

The literature on EDTP published in the past 2 years shows evidence that telepsychiatry implementation is associated with shorter wait times $[18 \bullet, 19,24]$, increased access [18•, $25 \bullet$, more discharges to home [21, 22], and increased sense of personal safety for providers [25•]. The receipt of EDTP is associated with lower usage of observation services for psychiatric visits, reducing the amount of time spent in the ED, and addressing the longstanding problem of ED crowding [22]. EDTP can also reduce ED LOS for pediatric patients and reduce travel for face-to-face evaluations, saving up to $2.22 \mathrm{~h}$ each day for on-call providers [18•]. Further, some providers for patients at risk for violence and behavioral dysregulation, for example, indicated a better feeling of personal safety with telepsychiatry [25•].

While the SCDMH providers reported seeing a slight decrease in virtual visits for the ED telepsychiatry program during the first quarter of 2020, available literature points to an increase in overall telehealth visits (i.e., mental health and non-mental health visits across all settings). Although we were not able to locate solid figures on the rates of ED telepsychiatry visits at a national level for comparison, we hypothesize that the increase seen in telehealth visits nationwide is mostly coming from "direct-to-consumer" visits, where patients are seen by the providers in their homes. On the contrary, the EDTP provides virtual care services to patients seeking in-person care at rural EDs. The availability of at-home mental health care via telehealth and fear of contracting COVID-19 in the ED setting could explain the dip in cases during the initial phases of the spread of COVID-19. At the same time, one key advantage of telepsychiatry highlighted by COVID-19 at the national level is alleviating current staffing burdens. This has been mirrored in the EDTP program in SC, where the availability of the EDTP infrastructure facilitated staffing rearrangements during COVID-19 and helped providers cope with the staffing challenges of the pandemic. 
Despite the success stories and documented value of EDTP, we would be remiss if we do not discuss some of the limitations of telepsychiatry programs. Technology disruptions, unequal availability in certain geographic areas, not suitable for all modes of treatment, not able to fully assess patients, and suboptimal for patients with certain diagnoses are some of those limitations. For example, frequent disruptions, difficulty reading non-verbal communications, and increased effort required to establish rapport have all been anecdotally reported in the literature [25•]. For many providers and patients, the sense of intimacy or provider-patient rapport during in-person consults may not be replicated on virtual platforms. Consultations that require non-verbal communications (e.g., insight-oriented/relational individual and group psychotherapy) or physical examination would require specific adaptation and adjustment to facilitate the treatment regimen [25•]. Providers have also discussed EDTP challenges for patients with specific conditions, such as auditory and/or visual impairments, migraine headaches, patients with paranoia, or other psychotic symptoms which may limit virtual interaction with the provider [25•].

Our review of the recent literature on telepsychiatry, in general, and EDTP specifically, amidst COVID-19 has identified several lessons and tips for successful and effective implementation. Some key elements include leadership and emotional support to help with morale and focus, daily email updates, regular meetings, technical tips and tricks, and technical support [25•]. Another key element of success is the need for an updated internet and sturdy technology (e.g., military-grade hand-held tablets with encrypted platforms) [19]. Further, when creating frameworks and programs around telepsychiatry, it is paramount to concurrently develop provider resources and trainings to support effective and efficient use of the equipment, as well as directories of mental health services in the patient's location [46, 47]. Using a continuous quality improvement cycle will also ensure that downfalls of the system are caught early and adjustments are made [47].

\section{Conclusions}

When considering the rise in use of telepsychiatry during the COVID-19 pandemic, the empirical evidence of this modality's efficacy, and South Carolina's success integrating EDTP in underserved areas, we foresee exponential growth in adoption of telepsychiatry services, both as a way to bridge gaps in access to care and as an emergency solution in times of major service disruption. Box 1 provides a summary of recommendations for systems interested in implementing telepsychiatry services in their practice.

Psychiatry is, by nature of the specialty, uniquely suited to virtual visits. Within the context of a public health emergency, the EDTP virtual delivery model decreases the likelihood that providers will be exposed to infections or natural hazards at work, which decreases disruption in practice and reduces the need for physical isolation or quarantine. This may help healthcare systems reduce burden on providers and staff. While a disruption of magnitude seen by COVID-19 may be infrequent, strategies used during the pandemic may be implemented to enhance care in rural or underserved settings, and/or enhance preparedness of communities and healthcare systems during more commonly occurring natural disasters. This is also true for otherwise healthy patients, particularly those presenting to EDs for mental health services, whether during a pandemic or outside a pandemic because it limits their exposure to undue burden of infections - a high risk in any ED setting.

One of the keys to South Carolina's success in treating psychiatric patients was the state-wide integrated system already in place which allowed us to perform telepsychiatry nearly seamlessly during the pandemic. We believe this technology is feasible, sustainable, and may enhance preparedness of healthcare systems to meet the needs of their patients. Telepsychiatry may also increase the public's confidence in the ability of the healthcare system to handle surges in service while abating fears of seeking care during disasters. In the future, public health messaging during emergencies that encourages people to seek care and combats fears of exposure or overburdening the healthcare system may be critical in ensuring timely access to crucial health services, including psychiatry.

\section{Box 1. Recommendations for Practice}

Based on our review we identified some practical recommendations for clinical practices interested in setting up or expanding telepsychiatry interventions, including:

Physical Setup

- Minimize distractions by choosing a quiet location away from street noise, pets, and other household members [25•].

- Consider using headphones to enhance patient privacy [25•].

Technical Considerations

- Use of integrated medical record system that allows for remote management of hospitalized patients [27].

- Look into the camera while speaking with the patient as opposed to looking at the screen [25•].

- Keep workspace simple by minimizing open windows, pop-up notifications, and alerts from phones or other applications $[25 \bullet]$. 


\section{Patient Considerations}

- Discuss patient expectations, including how the patient should alert provider if late to the virtual appointment, and whether patient will be expected to be seated for the session as opposed to lying down or driving [25•].

- Acknowledge the potential for discomfort during virtual care and create a backup plan for disruptions in technology. Acknowledge interruptions in service. [23•, 42]

\section{Staffing Considerations}

Maintain regular communication with peers in order to troubleshoot and share successes; embrace a culture of collaboration [25•, 27].

- Integrate planned breaks within your workday and take visual breaks from the computer screen [25•].

Availability of Data and Material Not applicable.

Code Availability Not applicable.

\section{Compliance with Ethical Standards}

Ethics Approval and Consent This article is a review of the current evidence based on published literature, so no IRB oversight was required. To help fill the current literature gap on ED telepsychiatry programs during COVID-19 and face validate our literature review findings, we had informal discussion with key stakeholders who consented to have their opinion and observations included in this review paper.

Competing Interests The authors declare no competing interests.

\section{References}

Papers of particular interest, published recently, have been highlighted as:

- Of importance

$\bullet$ Of major importance

1. Pesämaa L, Ebeling H, Kuusimäki ML, Winblad I, Isohanni M, Moilanen I. Videoconferencing in child and adolescent telepsychiatry: a systematic review of the literature. J Telemed Telecare. 2004;10(4):187-92.

2. Salmoiraghi A, Hussain S. A systematic review of the use of telepsychiatry in acute settings. Journal of Psychiatric Practice®. 2015 Sep 1;21(5):389-93.

3.•• Naslund JA, Mitchell LM, Joshi U, Nagda D, Lu C. Economic evaluation and costs of telepsychiatry programmes: a systematic review. Journal of telemedicine and telecare. $2020 \mathrm{Aug}$ 3:1357633X20938919. This systematic review reports on the cost effectiveness of telepsychiatry programs for mental healthcare.
4. Ward MM, Jaana M, Natafgi N. Systematic review of telemedicine applications in emergency rooms. Int J Med Informatics. 2015;84(9):601-16.

5. Yellowlees P, Burke MM, Marks SL, Hilty DM, Shore JH. Emergency telepsychiatry. J Telemed Telecare. 2008;14(6):277-81.

$6 . \bullet$ Reinhardt I, Gouzoulis-Mayfrank E, Zielasek J. Use of Telepsychiatry in emergency and crisis intervention: current evidence. Current Psychiatry Reports. 2019;21(8). This review article synthesizes literature on the use of telepsychiatry in mental crises or emergency situations published between 2014 and 2019.

7. Smith AC, Thomas E, Snoswell CL, Haydon H, Mehrotra A, Clemensen J, Caffery LJ. Telehealth for global emergencies: implications for coronavirus disease 2019 (COVID-19). J Telemed Telecare. 2020;26(5):309-13.

8. Leeb RT, Bitsko RH, Radhakrishnan L, Martinez P, Njai R, Holland KM. Mental health-related emergency department visits among children aged $<18$ years during the COVID19 pandemic - United States, January 1-October 17, 2020. MMWR Morb Mortal Wkly Rep. 2020;69(45):1675-80.

9. Castro VM, Perlis RH. Electronic health record documentation of psychiatric assessments in Massachusetts emergency department and outpatient settings during the coronavirus disease 2019 (COVID-19) Pandemic. JAMA Network Open. 2020;3(6).

10. Dragovic M, Pascu V, Hall T, Ingram J, Waters F. Emergency department mental health presentations before and during the COVID-19 outbreak in Western Australia. Australas Psychiatry. 2020;28(6):627-31.

11. Smalley CM, Malone DA, Meldon SW, Borden BL, Simon EL, Muir MKR, et al. The impact of COVID-19 on suicidal ideation and alcohol presentations to emergency departments in a large healthcare system. Am J Emerg Med. 2021;41:237-8.

12. Baugh JJ, White BA, McEvoy D, Yun BJ, Brown DFM, Raja AS, et al. The cases not seen: patterns of emergency department visits and procedures in the era of COVID-19. Am J Emerg Med. 2020.

13. Heppner Z, Shreffler J, Polites A, Ross A, Thomas JJ, Huecker M. COVID-19 and emergency department volume: the patients return but have different characteristics. Am J Emerg Med. 2020.

14. Goldenberg MN, Parwani V. Psychiatric emergency department volume during Covid-19 pandemic. Am J Emerg Med. 2021;41:233-4.

15. Hartnett KP, Kite-Powell A, DeVies J, Coletta MA, Boehmer TK, Adjemian J, et al. Impact of the COVID-19 pandemic on emergency department visits - United States, January 1, 2019-May 30, 2020. MMWR Morb Mortal Wkly Rep. 2020;69(23):699-704.

16. Gonçalves-Pinho M, Mota P, Ribeiro J, Macedo S, Freitas A. The impact of COVID-19 pandemic on psychiatric emergency department visits-a descriptive study. Psychiatr Q. 2020;25:1-1.

17.•• Freeman RE, Boggs KM, Zachrison KS, Freid RD, Sullivan AF, Espinola JA, et al. National Study of Telepsychiatry Use in U.S. Emergency Departments. Psychiatric Services. 2020;71(6):5406 . This study investigated the prevalence and applications of telepsychiatry in general EDs in the United States.

18. Reliford A, Adebanjo B. Use of telepsychiatry in pediatric emergency room to decrease length of stay for psychiatric patients, improve resident on-call burden, and reduce factors related to physician burnout. Telemedicine and e-Health. 2019;25(9):82832 . This study uses a prospective real-time survey of clinicians regarding child psychiatry fellow use of telepsychiatry.

19. Fairchild RM, Ferng-Kuo S-F, Laws S, Rahmouni H, Hardesty D. Telehealth decreases rural emergency department wait times for behavioral health patients in a group of critical access hospitals. Telemedicine and e-Health. 2019;25(12):1154-64. 
20. Maeng D, Richman JH, Lee HB, Hasselberg MJ. Impact of integrating psychiatric assessment officers via telepsychiatry on rural hospitals' emergency revisit rates. Journal of Psychosomatic Research. 2020;133:109997.

21. Kothadia RJ, Jones K, Saeed SA, Torres MJ. The impact of the North Carolina Statewide Telepsychiatry Program (NC-STeP) on patients' dispositions from emergency departments. Psychiatr Serv. 2020;71(12):1239-44.

22. Zhong C, Freeman RE, Boggs KM, Zachrison KS, Gao J, Espinola $\mathrm{JA}$, et al. Receipt of telepsychiatry and emergency department visit outcomes in New York state. Psychiatric Quarterly. 2021.

23. Roberge J, McWilliams A, Zhao J, Anderson WE, Hetherington $\mathrm{T}$, Zazzaro C, et al. Effect of a virtual patient navigation program on behavioral health admissions in the emergency department. JAMA Network Open. 2020;3(1). This clinical randomized trial assesses whether a behavioral health-virtual patient navigation program decreases hospitalization among patients presenting to the ED with a behavioral health crisis or need.

24. Brenner R, Madhusoodanan S, Logiudice J, et al. A comparison study of the turnaround time for telepsychiatry versus face-toface consultations in general hospital nonpsychiatric emergency rooms. Annals of Clinical Psychiatry : Official Journal of the American Academy of Clinical Psychiatrists. 2020;32(1):12-6.

25.• Chen JA, Chung W-J, Young SK, Tuttle MC, Collins MB, Darghouth SL, et al. COVID-19 and telepsychiatry: early outpatient experiences and implications for the future. Gen Hosp Psychiatry. 2020;66:89-95. This article describes primary barriers payment and regulatory challenges of widespread adoption of telepsychiatry prior to COVID-19.

26. Graell M, Morón-Nozaleda MG, Camarneiro R, Villaseñor Á, Yáñez S, Muñoz R, et al. Children and adolescents with eating disorders during COVID-19 confinement: difficulties and future challenges. Eur Eat Disord Rev. 2020;28(6):864-70.

27. Kalin ML, Garlow SJ, Thertus K, Peterson MJ. Rapid implementation of telehealth in hospital psychiatry in response to COVID19. Am J Psychiatry. 2020;177(7):636-7.

28. Fairchild RM, Ferng Kuo SF, Rahmouni H, Hardesty D. Telehealth increases access to care for children dealing with suicidality, depression, and anxiety in rural emergency departments. Telemedicine and e-Health. 2020;26(11):1353-62.

29. Fairchild R, Ferng Kuo SF, Rahmouni H, Hardesty D. An observational study of telemental care delivery and the context for involuntary commitment for mental health patients in a group of rural emergency departments. Telemedicine Reports. 2020;1(1):22-35.

30. Meyer JD, McKean AJ, Blegen RN, Demaerschalk BM. Emergency department telepsychiatry service model for a rural regional health system: the first steps. Telemedicine and e-Health. 2019;25(1):18-24.

31. Kroll DS, Stanghellini E, DesRoches SL, Lydon C, Webster A, O’Reilly M, Hurwitz S, Aylward PM, Cartright JA, McGrath EJ, Delaporta L. Virtual monitoring of suicide risk in the general hospital and emergency department. Gen Hosp Psychiatry. 2020;1(63):33-8.

32. Telepsychiatry Toolkit [Internet]. Telepsychiatry Toolkit Home. [cited 2021Apr19]. Available from: https://www.psychiatry.org/ psychiatrists/practice/telepsychiatry/toolkit
33. Bashshur RL, Shannon GW, Bashshur N, Yellowlees PM. The empirical evidence for telemedicine interventions in mental disorders. Telemed J E Health. 2016;22(2):87-113. https://doi.org/ 10.1089/tmj.2015.02064

34. Hubley S, Lynch SB, Schneck C, Thomas M, Shore J. Review of key telepsychiatry outcomes. World J Psychiatry. 2016;6(2):26982. https://doi.org/10.5498/wjp.v6.i2.269.

35. Shore $\mathrm{JH}$, Yellowlees $\mathrm{P}$, Caudill $\mathrm{R}$, et al. Best practices in videoconferencing-based telemental health April 2018. Telemed J E Health. 2018;24(11):827-32. https://doi.org/10.1089/tmj.2018. 0237.

36. Koonin LM, Hoots B, Tsang CA, Leroy Z, Farris K, Jolly B, et al. Trends in the use of telehealth during the emergence of the COVID-19 pandemic - United States, January-March 2020. MMWR Morb Mortal Wkly Rep. 2020;69(43):1595-9.

37. Demeke HB, Merali S, Marks S, Pao LZ, Romero L, Sandhu P, et al. Trends in use of telehealth among health centers during the COVID-19 pandemic - United States, June 26-November 6, 2020. MMWR Morb Mortal Wkly Rep. 2021;70(7):240-4.

38. Augusterfer EF, Mollica RF, Lavelle J. A review of telemental health in international and post-disaster settings. Int Rev Psychiatry. 2015;27(6):540-6. https://doi.org/10.3109/09540261.2015.108298.

39.• Severe J, Tang R, Horbatch F, Onishchenko R, Naini V, Blazek MC. Factors influencing patients' initial decisions regarding telepsychiatry participation during the COVID-19 pandemic: telephone-based survey. JMIR Form Res 2020;4(12):e25469. This study reports on patients' perception on the use of telepsychiatry during and post-COVID-19 in Michigan.

40. Bartels SJ, Baggett TP, Freudenreich O, Bird BL. COVID-19 emergency reforms in Massachusetts to support behavioral health care and reduce mortality of people with serious mental illness. Psychiatr Serv. 2020;71(10):1078-81.

41. Current state laws and reimbursement policies [Internet]. Cchpca. org. [cited 2021 Apr 19]. Available from: https://www.cchpca.org/ telehealth-policy/current-state-laws-and-reimbursement-policies

42. Morris NP, Hirschtritt ME. Telepsychiatry, hospitals, and the COVID-19 pandemic. Psychiatr Serv. 2020;71(12):1309-12.

43. Conrad RC, Baum ML, Shah SB, Levy-Carrick NC, Biswas J, Schmelzer NA, et al. Duties toward patients with psychiatric illness. Hastings Cent Rep. 2020;50(3):67-9.

44. Narasimhan M, Druss BG, Hockenberry JM, Royer J, Weiss P, Glick G, Marcus SC, Magill J. Impact of a telepsychiatry program at emergency departments statewide on the quality, utilization, and costs of mental health services. Psychiatr Serv. 2015;66(11):1167-72.

45. About DMH. 2021. [cited 2021Apr 19]. Available from: https:// scdmh.net/about/

46. Jobes DA, Crumlish JA, Evans AD. The COVID-19 pandemic and treating suicidal risk: the telepsychotherapy use of CAMS. J Psychother Integr. 2020;30(2):226-37.

47. Kroll DS, Shah SB, Gorman JM. The Joint Commission should reconsider its position on virtual monitoring of suicide risk. Gen Hosp Psychiatry. 2021;68:106.

Publisher's Note Springer Nature remains neutral with regard to jurisdictional claims in published maps and institutional affiliations. 